\title{
Influence of Housing Floor on Air Quality, Growth Traits, and Immunity in Broiler Chicken Farms
}

\author{
Essam S. Soliman ${ }^{*}$, Rania A. Hassan ${ }^{2}$
}

${ }^{1}$ Animal, Poultry and Environmental Hygiene Division, Department of Animal Hygiene, Zoonosis and Animal Behavior, Faculty of Veterinary Medicine, Suez Canal University, Ismailia 41522, Egypt; ${ }^{2}$ Animal Production Division, Department of Animal Wealth Development, Faculty of Veterinary Medicine, Suez Canal University, Ismailia 41522, Egypt.

\begin{abstract}
One of the challenges face the broiler industry is the choice of the floor system depending on its availability, cost, and ease of handling. The influence of different floors (wood shaving, rice husks, wheat straw, slats, and cages) on indoor air gases levels and microbial load, growth traits, carcass weights, some immune and edible organs' weights, antioxidant and cortisol levels, immunity, and intestinal microbiota in classic Hubbard broilers were investigated. A total of 200 one-day-old female classic Hubbard chicks were purchased and divided into five separate rooms. The $1^{\text {st }}$ room was supplied with wood shaving, the $2^{\text {nd }}$ with rice husks, the $3^{\text {rd }}$ with wheat straw, the $4^{\text {th }}$ with plastic slats, and the $5^{\text {th }}$ with horizontal cages. A total of 3705 samples including 525 air samples, 2100 environmental swabs (floors, walls, feeders, and waterers), 180 sera, 180 duodenal swabs, and 720 organs like liver, spleen, heart, and bursa of Fabricius were collected. The results revealed highly significant declines $(\mathrm{P}<0.01)$ in microclimatic carbon dioxide and ammonia concentrations, feed conversion ratios, cortisol hormone, total antioxidant capacity, lactate dehydrogenase, malondialdehyde, superoxide dismutase, and total bacterial and Enterobacteriaceae counts, as well as, highly significant increases $(\mathrm{P}<0.01)$ were recorded in weight gains, performance indices, live body weights, carcass weights, liver; spleen; heart and bursa of Fabricius weights, and immunoglobulin $G$ and $M$ concentrations in broilers raised in battery cages and on slat-floor. The study concluded that slatted floors and battery cages were able to maintain indoor air quality, reduce microbial contamination, and enhance growth traits and immunity of broiler chickens compared to traditional deep litter systems.
\end{abstract}

Keywords | Air quality, Broilers, Floor, Growth traits, Immunity.

Received | April 21, 2020; Accepted | June 21, 2020; Published | August 04, 2020

*Correspondence | Essam S Soliman, Animal, Poultry and Environmental Hygiene Division, Department of Animal Hygiene, Zoonosis and Animal Behavior,

Faculty of Veterinary Medicine, Suez Canal University, Ismailia 41522, Egypt; Email: soliman.essam@vet.suez.edu.eg

Citation | Soliman ES, Hassan RA (2020). Influence of housing floor on air quality, growth traits, and immunity in broiler chicken farms. Adv. Anim. Vet. Sci. 8(9): 997-1008.

DOI | http://dx.doi.org/10.17582/journal.aavs/2020/8.9.997.1008

ISSN (Online) | 2307-8316; ISSN (Print) | 2309-3331

Copyright (C) 2020 Soliman and Hassan. This is an open access article distributed under the Creative Commons Attribution License, which permits unrestricted use, distribution, and reproduction in any medium, provided the original work is properly cited.

\section{INTRODUCTION}

T he poultry industry as one of the main sources of animal protein and white meat in Egypt and worldwide faced a lot of obstacles, including the choice of the perfect housing floor system. Choosing floor system for raising broiler chickens relies on some factors such as; availability, economic cost, absorption capacity, maintenance and sustainability, possibilities of re-use, ease of handling, en- vironmental concern, and influence on performance and carcass yield (Garcia et al., 2012).

Over many years raising broiler chickens worldwide and in Egypt was depending on the deep litter system using a variety of litter types (Aviagen, 2016). Deep litter material like paper, sawdust, rice husks, wheat straw, and wood-shaving when used can be comfortable or barring enemy characteristics for broiler chickens. Using a deep 
litter system in raising broilers is dependent on many management factors to control litter moisture percent and $\mathrm{pH}$ as well as to control ammonia levels inside poultry farms (De Jong and Gunnink, 2014). If these managemental factors were not optimized and maintained, diseases risk like respiratory diseases and dermatitis can be increased (Sohirat Torfy et al., 2017; Petek et al., 2014). The degree of dryness of the bedding material in deep litter systems depends on many factors like stocking density, watering system, type of bedding material, indoor temperature, relative humidity, and degree of thermal insulation (Petek et al., 2015; Jacob et al., 2016).

Alternative methods for deep litter housing systems have been used for raising broiler chickens, including cages and slat. These methods have been widely used in the last twenty years over a large number of countries (Bilal et al., 2014). Battery and slatted floor systems had proven a lot of efficiency over the years in poultry raising and gained a lot of advantages like avoidance of litter problems, enhanced working conditions, ease of combating and eradication of infectious and contagious diseases. Later, the battery system was criticized from some points including the captivity of birds and their lack of natural behaviors, as well as, the development of some leg lesions (Özhan et al., 2016; Abreu et al., 2011), that is why slatted floor system gained more acceptance in raising broiler chicken compared to the battery system.

The current study was aiming to conduct a comparison between the different floor types (wood shaving, rice husks, wheat straw, slats, and cages) can be used locally in Egypt. The study included an analysis for the influence of these different floor systems on some parameters as; the indoor air gases (carbon dioxide and ammonia), air microbial load, growth traits, carcass weights, some immune and edible organs' weights, antioxidant levels (total antioxidant capacity, malondialdehyde, lactate dehydrogenase, and superoxide dismutase enzymes) and stress markers (cortisol hormone), immunity levels (immunoglobulin $\mathrm{G}$ and $\mathrm{M}$ concentrations), and intestinal microbiota (total bacterial and Enterobacteriaceae counts).

\section{MATERIALS AND METHODS}

\section{Experimental Birds Microclimate and Housing FLOORS}

A total of 200 one-day-old female classic Hubbard chicks were purchased from El-Helal Company. Birds were divided on their arrival into five separate rooms (five groups each 40 birds, with 4 replicates of ten birds). The rooms were supported with some protective measures to maintain an optimum biosecurity level as recommended by Soliman and Abdallah, (2020). These measures represented in re- stricted access to the rooms, foot dip at the entrance, restricted access to feed storage, fly and rodent-proof nets, prevent the attraction of wild birds, proper traffic control inside the rooms, and proper sanitation and disinfection program. The five rooms were supplied with different housing floors; the $1^{\text {st }}$ room was supplied with wood shaving litter, the $2^{\text {nd }}$ was supplied with rice husks litter, the $3^{\text {rd }}$ room was supplied with wheat straw litter, the $4^{\text {th }}$ room was supplied with plastic slats, and the $5^{\text {th }}$ room was supplied with horizontal cages. The floor of the $1^{\text {st }}, 2^{\text {nd }}$, and $3^{\text {rd }}$ rooms was treated previously before the application of different litter types and broiler's arrival with superphosphate 0.5 g. $\mathrm{m}^{-2}$ as recommended by Soliman et al. (2018) to prevent litter dampness, reduce moisture percent, reduce microbial activity, and minimize ammonia volatilization inside the rooms. The ventilation system in the five rooms was negative pressure cross-ventilation depending on suction fans on one sidewall and $\mathrm{V}$-shaped outlet windows on the other sidewall. Broilers in the five rooms were supplied with a continuous lighting regimen for 23 hours of lighting and one hour of darkness using blue LED lights as recommended by Soliman and Hassan, (2019).

Broilers were brooded on their arrival to the five rooms at a microclimatic temperature of $34^{\circ} \mathrm{C}$, optimized using oil and halogen heaters. Latter, the microclimatic temperature was manipulated by increasing the ventilation rates and lowering the heating hours to achieve a gradual decline of the temperature at a rate of $3.5^{\circ} \mathrm{C}$ weekly until achieving a stable $25^{\circ} \mathrm{C}$ by the end of the $3^{\text {rd }}$ week. Broilers were given permanent ad libitum access to water and corn-soybean basal ration to meet their basic nutritional requirements as recommended by National Research Council (NRC) (1994) as well as, Applegate and Angel, (2014) modifications. The ration constituted $23 \%$ protein, $5.6 \%$ fat, $3.80 \%$ crude fiber, and $2900 \mathrm{kcal} / \mathrm{kg}$ energy in the starter ration provided for the first fourteen days, and $21 \%$ protein, $2.8 \%$ fat, $3.39 \%$ crude fiber, and $3100 \mathrm{kcal} / \mathrm{kg}$ energy in the grower ration provided for the remaining period of the fattening cycle (25 days). Mortality rates, indoor and outdoor minimum, and maximum temperature and relative humidity were monitored and recorded daily during the experiment designed to last for 39 days. Broilers were subjected to an act of mass vaccination using de-chlorinated drinking water against infectious bronchitis using live attenuated virus vaccine (PESTIKAL B1 SPF H120 $\geq$ $\left.10^{3.5}\right)$ at $7^{\text {th }}$ day; infectious bursal disease using live attenuated virus vaccine (SER-VAC D78 Strain VMG91 $\geq 10^{3.0}$ ) at $14^{\text {th }}$ and $21^{\text {st }}$ days, and Newcastle virus disease using live lentogenic virus vaccine (PESTIKAL Lasota $\geq 10^{6.0}$ ) at $16^{\text {th }}$ and $26^{\text {th }}$ days.

\section{Performance Indices}

Average live body weights (LBW/g) were estimated week- 
ly by weighing a representative number of birds (approximately 36 birds) in each group. The number of weighed birds was calculated using simple random sampling design according to Thrusfield (2005) with an expected error 5\% using the following formula:

\section{$\mathrm{n}=1.96^{2} \mathrm{P}_{\exp }\left(1-\mathrm{P}_{\exp }\right) / \mathrm{d}^{2}$}

Where $\mathrm{n}=$ required sample size, Pexp = expected prevalence, $\mathrm{d}=$ desired absolute precision. Feed intakes $(\mathrm{FI} / \mathrm{g})$ were calculated by diving the total amount of ration consumed in each room by the total number of viable birds in the room. Bodyweight gains (WG/g), feed conversion ratios (FCR \%), and performance indices (PI) were calculated as recommended by Soliman and Hassan, (2017).

\section{SAMPLING}

A total of 3705 samples including 525 air samples (three sample daily in each room for five weeks), 2100 environmental swabs (floors, walls, feeders, and waterers, they were collected by a rate of 3 swabs daily per type in each room for five weeks), 180 sera, 180 duodenal swabs, and 720 organs (180 each) like liver, spleen, heart, and bursa of Fabricius were collected by the end of the experiment. Air samples were collected using air canister for passive sampling of air and were later passed on $9 \mathrm{ml}$ buffered peptone water in the laboratory for further analysis (chemical and bacteriological). Environmental and non-environmental swabs were collected on $9 \mathrm{ml}$ buffered peptone water and transferred to the laboratory in a dry ice box within 2-3 hours to be examined.

A total of 180 birds (36 birds from each room) were sacrificed by the end of the experiment to collect blood samples, birds were de-feathered, weighed, and expressed by grams (carcass weight; CW/g). Edible and immune organs like liver, spleen, heart, and bursa of Fabricius were collected, weighed, and expressed per grams. Sacrificed birds were hygienically disposed of after sampling using a burial method with the lining of the burial bits with lime. Blood samples after collection were kept in a water bath at $25^{\circ} \mathrm{C}$ for 30 minutes and centrifuged at $3500 \mathrm{rpm}$ for $20 \mathrm{~min}-$ utes. None hemolyzed sera were collected in Eppendorf tubes and stored at $-20{ }^{\circ} \mathrm{C}$ until tested for cortisol level, antioxidant markers, and immunological assay (Soliman et al., 2017).

\section{IndOOR Air QUALITY}

Air samples (three samples daily in each room for five weeks) were examined for the levels of carbon dioxide $\left(\mathrm{CO}_{2}\right)$ and ammonia $\left(\mathrm{NH}_{3}\right)$ using potentiometric titration against oxalic acid and ammonium hydroxide respectively as recommended by APHA (2017). The calculated results were compared and confirmed by the readings obtained from inside the experimental rooms during the fattening cycle using digital carbon dioxide meter (e Top $77535 \mathrm{Dig}-$ ital 3-in-1 $\mathrm{CO}_{2}$ 0- 9999 ppm, Temperature DP WB RH Humidity IAQ Air Quality Meter Tester Monitor Carbon) and ammonia meter (Digital Portable $\mathrm{NH}_{3}$ Meter Ammonia Gas Detector 0: 100 ppm).

\section{Antioxidant and Stress Markers}

Sera samples (180 samples collected via sacrificing 36 birds from each room) were examined for antioxidant markers like total antioxidant capacity (TAC), lactate dehydrogenase (LDH), malondialdehyde (MDA), and superoxide dismutase (SOD) calorimetrically using ROCHE COBAS Integra 800 chemical analyzer. Cortisol hormone as well as, immunoglobulin IgG and IgM concentrations were measured by using ROCHE Elecsys 1010 Immunoassay Analyzer (Wu et al., 2017).

\section{Bacteriological Examination}

Air samples (525 samples), environmental swabs (floors, walls, feeders, and waterers, 2100 samples), and non-environmental swabs (180 duodenal swabs) collected on $9 \mathrm{ml}$ buffered peptone water were prepared as recommended by APHA (2012). Tenfold serial dilutions up to $10^{-10}$ were prepared.

Total bacterial (TBC) and total Enterobacteriaceae counts (TEC) were carried out using a drop plate as recommended by Soliman et al., (2016) and Kim and Lee, (2016). Total bacterial counts were performed onto standard plate count agar (PCA, LAB-M, LAB149; 500g) at $37^{\circ} \mathrm{C}$ for $24-48 \mathrm{~h}$. Meanwhile, TEC was conducted using Eosin Methylene Blue Agar (EMB, LAB-M, LAB061; 500g ) at $37^{\circ} \mathrm{C}$ for 24 - 48 h. Plates were counted using the Dark-field colony counter ((R164109 Reichert-Jung Quebec Darkfield 3325 Colony Counter) (Murray et al., 2015).

\section{Statistical Analysis}

Statistical analysis was carried out using the statistical package for social sciences (SPSS version 20.0, 2016). The raw data were analyzed statistically using multifactorial Analysis of Variance (Two-Way ANOVA) to determine the overall influence of the housing floors and broiler's age and their interactions. The statistical model empathized as follow:

$Y_{i j k}=\mu+\alpha_{i}+\beta_{j}+(\alpha \beta)_{i j}+\varepsilon_{i j k}$

Where $Y_{\mathrm{ijk}}$ was the measurement of dependent variables; $\mu$ was overall mean; $\alpha_{i}$ was the fixed effect of the floor systems, $\beta_{\mathrm{i}}$ was the fixed effect of broiler's age, $(\alpha \beta)_{\mathrm{ij}}$ was the interactions of housing floor systems by broiler's age, and $\varepsilon_{\mathrm{ijk}}$ was the random error. Total bacterial and Enterobacteriaceae counts of environmental and non-environmental samples were transformed and expressed as logarithms 
Table 1: Microclimatic indoor gas levels (Mean \pm SE) inside broiler rooms of different housing floor systems.

\begin{tabular}{|c|c|c|c|}
\hline Floor system & Age /weeks & $\mathrm{CO}_{2} / \mathrm{ppm}$ & $\mathrm{NH}_{3} / \mathrm{ppm}$ \\
\hline \multicolumn{4}{|c|}{ Overall means among different floor systems } \\
\hline \multicolumn{2}{|l|}{ Wood shaving } & $14710^{a} \pm 10.62$ & $26.2^{\mathrm{a}} \pm 0.03$ \\
\hline \multicolumn{2}{|l|}{ Rice husks } & $14190^{b} \pm 11.66$ & $19.4^{b} \pm 0.01$ \\
\hline \multicolumn{2}{|l|}{ Wheat straw } & $13030^{c} \pm 18.47$ & $16.1^{\mathrm{c} \pm 0.12}$ \\
\hline \multicolumn{2}{|l|}{ Slats } & $2640^{\mathrm{d}} \pm 11.89$ & $4.1^{\mathrm{e}} \pm 0.01$ \\
\hline \multicolumn{2}{|l|}{ Battery } & $2170^{\mathrm{e}} \pm 7.56$ & $4.9^{\mathrm{d}} \pm 0.03$ \\
\hline \multicolumn{2}{|l|}{$\mathrm{P}$ value } & 0.000 & 0.001 \\
\hline \multicolumn{4}{|c|}{ Overall means among different broiler ages } \\
\hline \multicolumn{2}{|l|}{$1^{\text {st }}$ week } & $5110^{\mathrm{e}} \pm 16.25$ & $9.8^{\mathrm{e}} \pm 0.02$ \\
\hline \multicolumn{2}{|l|}{$2^{\text {nd }}$ week } & $6950^{\mathrm{d}} \pm 13.44$ & $11.1^{\mathrm{d}} \pm 0.03$ \\
\hline \multicolumn{2}{|l|}{$3^{\text {rd }}$ week } & $9880^{c} \pm 23.15$ & $13.9^{\mathrm{c}} \pm 0.04$ \\
\hline \multicolumn{2}{|l|}{$4^{\text {th }}$ week } & $12070^{\mathrm{b}} \pm 18.95$ & $16.4^{\mathrm{b}} \pm 0.01$ \\
\hline \multicolumn{2}{|l|}{$5^{\text {th }}$ week } & $12730^{\mathrm{a}} \pm 17.55$ & $19.5^{\mathrm{a}} \pm 0.01$ \\
\hline \multicolumn{2}{|l|}{$P$ value } & 0.001 & 0.000 \\
\hline \multicolumn{4}{|c|}{ Floor systems versus broiler age interactions } \\
\hline \multirow[t]{5}{*}{ Wood shaving } & $1^{\text {st }}$ week & $7900^{\mathrm{e}} \pm 44.94$ & $19.2^{\mathrm{e}} \pm 0.13$ \\
\hline & $2^{\text {nd }}$ week & $11100^{\mathrm{d}} \pm 45.29$ & $21.1^{\mathrm{d}} \pm 0.28$ \\
\hline & $3^{\text {rd }}$ week & $15900^{c} \pm 20.16$ & $26.3^{c} \pm 0.18$ \\
\hline & $4^{\text {th }}$ week & $19200^{\mathrm{b}} \pm 15.27$ & $29.8^{\mathrm{b}} \pm 0.13$ \\
\hline & $5^{\text {th }}$ week & $19450^{\mathrm{a}} \pm 9.75$ & $34.6^{a} \pm 0.13$ \\
\hline \multirow[t]{5}{*}{ Rice husks } & $1^{\text {st }}$ week & $8050^{\mathrm{e}} \pm 47.04$ & $13.6^{\mathrm{e}} \pm 0.26$ \\
\hline & $2^{\text {nd }}$ week & $10400^{\mathrm{d}} \pm 25.60$ & $15.6^{\mathrm{d}} \pm 0.16$ \\
\hline & $3^{\text {rd }}$ week & $14350^{c} \pm 35.78$ & $19.2^{c} \pm 0.11$ \\
\hline & $4^{\text {th }}$ week & $18550^{\mathrm{b}} \pm 36.09$ & $22.2^{b} \pm 0.13$ \\
\hline & $5^{\text {th }}$ week & $19600^{\mathrm{a}} \pm 22.11$ & $26.4^{a} \pm 0.22$ \\
\hline \multirow[t]{5}{*}{ Wheat straw } & $1^{\text {st }}$ week & $6900^{e} \pm 14.52$ & $10.4^{e} \pm 0.17$ \\
\hline & $2^{\text {nd }}$ week & $9300^{\mathrm{d}} \pm 26.35$ & $12.6^{\mathrm{d}} \pm 0.29$ \\
\hline & $3^{\text {rd }}$ week & $12900^{c} \pm 39.29$ & $16.1^{\mathrm{c}} \pm 0.22$ \\
\hline & $4^{\text {th }}$ week & $17400^{\mathrm{b}} \pm 20.81$ & $18.9^{b} \pm 0.30$ \\
\hline & $5^{\text {th }}$ week & $18650^{\mathrm{a}} \pm 45.36$ & $22.7^{\mathrm{a}} \pm 0.37$ \\
\hline \multirow[t]{5}{*}{ Slats } & $1^{\text {st }}$ week & $1550^{\mathrm{e}} \pm 13.84$ & $3.6^{\mathrm{d} \pm 0.01}$ \\
\hline & $2^{\text {nd }}$ week & $2150^{\mathrm{d}} \pm 17.37$ & $3.3^{\mathrm{e}} \pm 0.06$ \\
\hline & $3^{\text {rd }}$ week & $3250^{\mathrm{b}} \pm 11.18$ & $3.9^{c} \pm 0.05$ \\
\hline & $4^{\text {th }}$ week & $2900^{c} \pm 16.66$ & $4.3^{\mathrm{b}} \pm 0.09$ \\
\hline & $5^{\text {th }}$ week & $3350^{\mathrm{a}} \pm 16.37$ & $5.3^{a} \pm 0.08$ \\
\hline \multirow[t]{5}{*}{ Battery } & $1^{\text {st }}$ week & $1150^{\mathrm{e}} \pm 10.69$ & $2.3^{\mathrm{e}} \pm 0.01$ \\
\hline & $2^{\text {nd }}$ week & $1800^{\mathrm{d}} \pm 18.64$ & $3.2^{\mathrm{d} \pm 0.03}$ \\
\hline & $3^{\text {rd }}$ week & $3000^{\mathrm{a}} \pm 12.90$ & $4.1^{\mathrm{c}} \pm 0.09$ \\
\hline & $4^{\text {th }}$ week & $2300^{c} \pm 18.64$ & $6.6^{b} \pm 0.02$ \\
\hline & $5^{\text {th }}$ week & $2600^{b} \pm 16.67$ & $8.4^{a} \pm 0.06$ \\
\hline \multicolumn{2}{|l|}{$P$ value } & 0.000 & 0.000 \\
\hline
\end{tabular}

Means carrying different superscripts in the same column are significantly different at $(\mathrm{P} \leq 0.05)$ or highly significantly different at $(\mathrm{P}<0.01)$. Means carrying the same superscripts in the same column are non-significantly different at $(\mathrm{P}<0.05)$.

$\mathrm{CO}_{2}=$ Carbon dioxide, $\mathrm{NH}_{3}=$ Ammonia, $\mathrm{SE}=$ Standard error. 
using Microsoft Excel 2016. The results were expressed as highly significant at $(p \leq 0.01)$, significant at $(p \leq 0.05)$, and non-significant at $(\mathrm{p}>0.05)$.

\section{RESULTS}

\section{Microclimatic Gases LeVels}

Indoor air gases in Table 1 reflected synchronized highly significant declines $(\mathrm{P}<0.01)$ to normal thresholds of the microclimatic levels of both $\mathrm{CO}_{2}$ and $\mathrm{NH}_{3}$ gases of rooms in which broilers were raised in the battery system and on slatted floor, respectively, rather than deep litters based on wood shaving, rice husks, and wheat straw. From a normal perspective, the microclimatic levels of $\mathrm{CO}_{2}$ and $\mathrm{NH}_{3}$ were increasing as broiler chickens grow and get older.

\section{Growth Traits}

Bodyweight gains (WG) and performance indices (PI), as recorded in Table 2 revealed highly significant increases (P $<0.01$ ) in broilers raised on the battery and slatted floor with no significant differences between the battery and slatted floor systems in WG values. Feed conversion ratios $(\mathrm{FCR})$ reflected highly significant declines $(\mathrm{P}<0.01)$ in broilers raised in the battery system and on the slatted floor with no significant differences between each other. Feed intakes (FI) revealed highly significant increases $(\mathrm{P}<$ $0.01)$ in broilers raised on the slatted floor and highly significant declines $(\mathrm{P}<0.01)$ in broilers raised in the battery system. Meanwhile, water intake- WI (Table 1) revealed no significant differences between all the floor systems used in the experiment.

The overall means of growth traits among different broiler ages (Table 2) revealed highly significant increases $(\mathrm{P}<$ $0.01)$ of WG $(\mathrm{g})$ during the $4^{\text {th }}, 3^{\text {rd }}, 5^{\text {th }}, 2^{\text {nd }}$, and $1^{\text {st }}$ weeks, respectively, highly significant declines $(\mathrm{P}<0.01)$ of FCR (\%) during the $1^{\text {st }}, 4^{\text {th }}, 3^{\text {rd }}, 2^{\text {nd }}$, and $5^{\text {th }}$ weeks, respectively, highly significant increases $(\mathrm{P}<0.01)$ of $\mathrm{PI}$ during the $4^{\text {th }}$, $5^{\text {th }}, 3^{\text {rd }}, 2^{\text {nd }}$, and $1^{\text {st }}$ weeks, respectively, highly significant increases $(\mathrm{P}<0.01)$ of $\mathrm{FI}$ and WI during the $5^{\text {th }}, 4^{\text {th }}, 3^{\text {rd }}, 2^{\text {nd }}$, and $1^{\text {st }}$ weeks, respectively, and highly significant increases $(\mathrm{P}<0.01)$ of WI/FI ratios during the $5^{\text {th }}, 4^{\text {th }}, 1^{\text {st }}, 3^{\text {rd }}$, and $2^{\text {nd }}$ weeks, respectively.

\section{Live Weight and Carcass Quality}

Live body weights revealed highly significant increases ( $\mathrm{P}$ $<0.01$ ), as recorded in Table 3, in broilers raised in the battery system and on the slatted floor compared to deep litters with no significant differences in the live body weights between broilers raised on rice husks and wood-shaving floor systems. Carcass weights revealed highly significant increases $(\mathrm{P}<0.01)$ in broilers raised in the battery system and on the slatted floor compared to deep litters with no significant differences in the carcass weights between broilers raised on rice husks and wheat straw floor systems (Table 3).

Liver weights revealed highly significant increases $(\mathrm{P}<$ 0.01 ), in Table 3 , in broilers raised in the battery system and on the slatted floor compared to deep litters with no significant differences in the liver weights between broilers raised on wheat straw and wood-shaving floor systems. While, spleen; heart and bursa of Fabricius revealed highly significant increases $(P<0.01)$ in broilers raised in the battery system and on the slatted floor compared to deep litters with no significant differences in the spleen; heart and bursa of Fabricius between broilers raised on all deep litter systems (Table 3).

\section{Stress And Antioxidant Markers}

Serum cortisol concentrations revealed highly significant declines $(\mathrm{p}<0.01)$, as recorded in Table 4 , in broilers raised in the battery system and on the slatted floor compared to deep litters with no significant differences in the cortisol levels between broilers raised in the battery system and on slatted floor.

Malondialdehyde revealed in Table 4, highly significant declines $(\mathrm{P}<0.01)$ in broilers raised in the battery system, on slatted, and wheat straw floors compared to rice husks and wood-shaving litter system. Meanwhile, total antioxidant capacity, lactate dehydrogenase enzyme, and superoxide dismutase recorded highly significant declines $(\mathrm{P}<$ 0.01 ) in broilers raised in the battery system and on the slatted floor compared to deep litters (Table 4).

\section{IMMUNOGLOBULIN}

\section{Concentrations}

AND

\section{Microbial Loads}

Highly significant increases $(\mathrm{P}<0.01)$ were recorded ( $\mathrm{Ta}-$ ble 5) in serum concentrations of total immunoglobulin $\operatorname{IgG}$ and $\operatorname{IgM}$ in broilers raised in the battery system and on the slatted floor compared to the other tested floor systems.

Highly significant declines $(\mathrm{P}<0.01)$ in Table 6 were recorded in total bacterial counts of intestinal (duodenal) swabs in broilers raised in battery and on slatted floors with no significant differences in total bacterial counts of intestinal (duodenal) swabs between slatted and rice husks floors, in total bacterial counts of floor in battery and on slatted floors with no significant differences in total bacterial counts of slats and wheat straw, in total bacterial counts of wall swabs in battery and on slatted floors, in total bacterial counts of feeder swabs in battery and on slatted floors with no significant differences in total bacterial counts of wheat straw and wood-shaving, in total bacterial counts of waterer swabs in battery and on slatted floors with no significant differences in total bacterial counts of wood 
Table 2: Performance traits (Mean $\pm \mathrm{SE}$ ) of broilers raised on different housing floor systems.

\begin{tabular}{lllllll}
$\begin{array}{l}\text { Floor } \\
\text { system Age / } \\
\text { week }\end{array}$ & BWG / g & FCR / $\%$ & PI & FI / g & WI/ ml & WI/FI \% \\
Overall means among different floor systems & & & & \\
\hline Wood shaving & $311.6^{\mathrm{c}} \pm 1.23$ & $1.35^{\mathrm{c}} \pm 0.01$ & $5.91^{\mathrm{d}} \pm 0.02$ & $443.8^{\mathrm{d}} \pm 1.31$ & $846^{\mathrm{a}} \pm 1.83$ & $1.68^{\mathrm{a}} \pm 0.01$ \\
\hline Rice husks & $320.2^{\mathrm{c}} \pm 3.13$ & $1.64^{\mathrm{a}} \pm 0.01$ & $5.55^{\mathrm{a}} \pm 0.05$ & $498.0^{\mathrm{b}} \pm 2.10$ & $838^{\mathrm{a}} \pm 1.22$ & $1.63^{\mathrm{a}} \pm 0.02$ \\
\hline Wheat straw & $351.5^{\mathrm{b}} \pm 2.11$ & $1.48^{\mathrm{b}} \pm 0.02$ & $6.55^{\mathrm{c}} \pm 0.12$ & $496.9^{\mathrm{b}} \pm 2.52$ & $841^{\mathrm{a}} \pm 2.11$ & $1.62^{\mathrm{a}} \pm 0.01$ \\
\hline Slats & $401.9^{\mathrm{a}} \pm 1.09$ & $1.29^{\mathrm{d}} \pm 0.01$ & $7.98^{\mathrm{b}} \pm 0.09$ & $509.2^{\mathrm{a}} \pm 1.15$ & $869^{\mathrm{a}} \pm 1.54$ & $1.62^{\mathrm{a}} \pm 0.03$ \\
Battery & $408.5^{\mathrm{a}} \pm 2.18$ & $1.13^{\mathrm{d}} \pm 0.01$ & $8.50^{\mathrm{a}} \pm 0.07$ & $486.6^{\mathrm{c}} \pm 1.56$ & $828^{\mathrm{a}} \pm 1.33$ & $1.64^{\mathrm{a}} \pm 0.03$ \\
\hline P value & 0.000 & 0.000 & 0.000 & 0.008 & 0.589 & 0.667
\end{tabular}

Overall means among different broiler ages

\begin{tabular}{lllllll}
$1^{\text {st }}$ week & $100.6^{\mathrm{e}} \pm 1.12$ & $1.11^{\mathrm{d}} \pm 0.01$ & $1.43^{\mathrm{e}} \pm 0.12$ & $107.4^{\mathrm{e}} \pm 1.55$ & $188^{\mathrm{e}} \pm 1.33$ & $1.75^{\mathrm{b}} \pm 0.02$ \\
$2^{\text {nd }}$ week & $221.8^{\mathrm{d}} \pm 1.22$ & $1.56^{\mathrm{b}} \pm 0.02$ & $2.41^{\mathrm{d}} \pm 0.08$ & $345.8^{\mathrm{d}} \pm 1.74$ & $381^{\mathrm{d}} \pm 1.38$ & $1.11^{\mathrm{d} \pm 0.00}$ \\
$3^{\text {rd }}$ week & $462.3^{\mathrm{b}} \pm 1.13$ & $1.20^{\mathrm{c}} \pm 0.02$ & $7.23^{\mathrm{c}} \pm 0.03$ & $551.2^{\mathrm{c}} \pm 2.11$ & $651^{\mathrm{c}} \pm 2.53$ & $1.21^{\mathrm{c}} \pm 0.01$ \\
$4^{\text {th }}$ week & $599.3^{\mathrm{a}} \pm 1.15$ & $1.11^{\mathrm{d}} \pm 0.03$ & $13.65^{\mathrm{a}} \pm 0.01$ & $637.1^{\mathrm{b}} \pm 1.87$ & $1145^{\mathrm{b}} \pm 1.42$ & $1.80^{\mathrm{b}} \pm 0.02$ \\
$5^{\text {th }}$ week & $409.6^{\mathrm{c}} \pm 2.11$ & $2.09^{\mathrm{a}} \pm 0.01$ & $9.77^{\mathrm{b}} \pm 0.02$ & $792.8^{\mathrm{a}} \pm 2.13$ & $1857^{\mathrm{a}} \pm 1.22$ & $2.34^{\mathrm{a}} \pm 0.02$ \\
\hline P value & 0.000 & 0.000 & 0.000 & 0.000 & 0.000 & 0.001
\end{tabular}

\section{Floor systems versus broiler age interactions}

\begin{tabular}{|c|c|c|c|c|c|c|c|}
\hline \multirow{5}{*}{$\begin{array}{l}\text { Wood } \\
\text { shaving }\end{array}$} & $1^{\text {st }}$ & $121.9^{\mathrm{e}} \pm 1.02$ & $0.87^{\mathrm{d}} \pm 0.01$ & $1.95^{\mathrm{d}} \pm 0.03$ & $106.2^{\mathrm{e}} \pm 0.79$ & $126^{\mathrm{e}} \pm 1.04$ & $1.19^{\mathrm{e}} \pm 0.01$ \\
\hline & $2^{\text {nd }}$ & $213.2^{\mathrm{d}} \pm 2.44$ & $1.29^{\mathrm{c}} \pm 0.01$ & $2.95^{\mathrm{c}} \pm 0.04$ & $276.6^{\mathrm{d}} \pm 0.43$ & $349^{\mathrm{d}} \pm 2.36$ & $1.26^{\mathrm{d}} \pm 0.08$ \\
\hline & $3^{\text {rd }}$ & $449.7^{\mathrm{a}} \pm 2.04$ & $0.95^{\mathrm{d}} \pm 0.05$ & $9.15^{a} \pm 0.07$ & $429.2^{\mathrm{c}} \pm 2.49$ & $651^{c} \pm 2.97$ & $1.58^{c} \pm 0.12$ \\
\hline & $4^{\text {th }}$ & $384.6^{c} \pm 2.48$ & $1.61^{\mathrm{b}} \pm 0.02$ & $7.55^{\mathrm{b}} \pm 0.09$ & $620.1^{\mathrm{b}} \pm 5.23$ & $1297^{\mathrm{b}} \pm 2.66$ & $2.09^{b} \pm 0.05$ \\
\hline & $5^{\text {th }}$ & $388.8^{\mathrm{b}} \pm 1.50$ & $2.02^{\mathrm{a}} \pm 0.00$ & $7.93^{b} \pm 0.03$ & $786.7^{\mathrm{a}} \pm 1.29$ & $1806^{a} \pm 3.49$ & $2.29^{a} \pm 0.01$ \\
\hline \multirow{5}{*}{$\begin{array}{l}\text { Rice } \\
\text { husks }\end{array}$} & $1^{\text {st }}$ & $98.1^{\mathrm{e}} \pm 1.05$ & $1.08^{\mathrm{d}} \pm 0.00$ & $1.37^{\mathrm{d}} \pm 0.01$ & $106.3^{\mathrm{e}} \pm 0.46$ & $210^{\mathrm{e}} \pm 1.37$ & $1.97^{\mathrm{b}} \pm 0.01$ \\
\hline & $2^{\text {nd }}$ & $228.3^{\mathrm{d}} \pm 1.94$ & $1.58^{b} \pm 0.01$ & $2.38^{\mathrm{c}} \pm 0.03$ & $361.4^{\mathrm{d}} \pm 0.30$ & $387^{\mathrm{d}} \pm 1.96$ & $1.07^{\mathrm{d}} \pm 0.01$ \\
\hline & $3^{\text {rd }}$ & $411.8^{\mathrm{b}} \pm 2.31$ & $1.38^{\mathrm{c}} \pm 0.01$ & $5.70^{\mathrm{b}} \pm 0.04$ & $570.2^{\mathrm{c}} \pm 1.78$ & $640^{c} \pm 1.58$ & $1.12^{\mathrm{d}} \pm 0.01$ \\
\hline & $4^{\text {th }}$ & $605.4^{a} \pm 2.86$ & $1.07^{\mathrm{d}} \pm 0.01$ & $12.98^{\mathrm{a}} \pm 0.07$ & $650.3^{b} \pm 2.13$ & $1125^{\mathrm{b}} \pm 1.03$ & $1.73^{c} \pm 0.15$ \\
\hline & $5^{\text {th }}$ & $257.5^{\mathrm{c}} \pm 2.17$ & $3.11^{\mathrm{a}} \pm 0.02$ & $5.30^{\mathrm{b}} \pm 0.05$ & $801.7^{\mathrm{a}} \pm 0.71$ & $1830^{a} \pm 5.72$ & $2.28^{\mathrm{a}} \pm 0.01$ \\
\hline \multirow{5}{*}{$\begin{array}{l}\text { Wheat } \\
\text { straw }\end{array}$} & $1^{\mathrm{st}}$ & $107.4^{\mathrm{e}} \pm 1.83$ & $1.01^{\mathrm{d}} \pm 0.01$ & $1.54^{\mathrm{d}} \pm 0.04$ & $108.8^{\mathrm{e}} \pm 0.68$ & $197^{\mathrm{e}} \pm 2.02$ & $1.81^{\mathrm{b}} \pm 0.01$ \\
\hline & $2^{\text {nd }}$ & $199.0^{\mathrm{d}} \pm 3.08$ & $1.81^{b} \pm 0.02$ & $1.95^{\mathrm{d}} \pm 0.03$ & $361.0^{\mathrm{d}} \pm 0.42$ & $389^{\mathrm{d}} \pm 2.07$ & $1.08^{\mathrm{d}} \pm 0.02$ \\
\hline & $3^{\text {rd }}$ & $425.5^{\mathrm{b}} \pm 1.53$ & $1.42^{c} \pm 0.01$ & $5.49^{c} \pm 0.02$ & $604.6^{c} \pm 0.71$ & $679^{c} \pm 3.21$ & $1.12^{\mathrm{d}} \pm 0.01$ \\
\hline & $4^{\text {th }}$ & $681.7^{\mathrm{a}} \pm 1.66$ & $0.92^{\mathrm{d}} \pm 0.00$ & $15.77^{a} \pm 0.07$ & $631.8^{\mathrm{b}} \pm 3.16$ & $1075^{\mathrm{b}} \pm 4.65$ & $1.70^{c} \pm 0.14$ \\
\hline & $5^{\text {th }}$ & $344.0^{c} \pm 2.33$ & $2.26^{a} \pm 0.01$ & $7.98^{b} \pm 0.05$ & $778.2^{\mathrm{a}} \pm 0.86$ & $1864^{a} \pm 1.28$ & $2.39^{a} \pm 0.01$ \\
\hline \multirow[t]{5}{*}{ Slats } & $1^{\mathrm{st}}$ & $106.7^{\mathrm{e}} \pm 2.18$ & $1.01^{\mathrm{c}} \pm 0.02$ & $1.51^{\mathrm{e}} \pm 0.05$ & $107.5^{\mathrm{e}} \pm 0.34$ & $195^{\mathrm{e}} \pm 2.72$ & $1.82^{\mathrm{b}} \pm 0.02$ \\
\hline & $2^{\text {nd }}$ & $229.5^{\mathrm{d}} \pm 3.31$ & $1.62^{\mathrm{a}} \pm 0.02$ & $2.34^{\mathrm{d}} \pm 0.03$ & $372.3^{\mathrm{d}} \pm 0.59$ & $412^{\mathrm{d}} \pm 5.51$ & $1.10^{c} \pm 0.01$ \\
\hline & $3^{\text {rd }}$ & $467.9^{c} \pm 1.56$ & $1.25^{\mathrm{b}} \pm 0.01$ & $6.76^{c} \pm 0.04$ & $587.2^{\mathrm{c}} \pm 2.92$ & $632^{c} \pm 4.64$ & $1.07^{\complement} \pm 0.01$ \\
\hline & $4^{\text {th }}$ & $714.4^{a} \pm 1.93$ & $0.91^{\mathrm{d}} \pm 0.00$ & $17.07^{a} \pm 0.07$ & $654.0^{\mathrm{b}} \pm 1.10$ & $1173^{\mathrm{b}} \pm 1.07$ & $1.79^{\mathrm{b}} \pm 0.16$ \\
\hline & $5^{\text {th }}$ & $491.3^{b} \pm 3.96$ & $1.68^{a} \pm 0.01$ & $12.23^{b} \pm 0.11$ & $824.9^{\mathrm{a}} \pm 1.05$ & $1934^{\mathrm{a}} \pm 1.19$ & $2.34^{a} \pm 0.01$ \\
\hline \multirow[t]{5}{*}{ Battery } & $1^{\mathrm{st}}$ & $69.1^{\mathrm{e}} \pm 3.69$ & $1.60^{\mathrm{a}} \pm 0.08$ & $0.76^{\mathrm{e}} \pm 0.06$ & $108.4^{\mathrm{e}} \pm 0.37$ & $211^{\mathrm{e}} \pm 5.54$ & $1.94^{\mathrm{b}} \pm 0.04$ \\
\hline & $2^{\text {nd }}$ & $239.4^{\mathrm{d}} \pm 1.34$ & $1.51^{a} \pm 0.06$ & $2.42^{\mathrm{d}} \pm 0.15$ & $357.9^{\mathrm{d}} \pm 4.68$ & $367^{\mathrm{d}} \pm 5.25$ & $1.02^{\mathrm{e}} \pm 0.01$ \\
\hline & $3^{\text {rd }}$ & $556.7^{\mathrm{c}} \pm 1.50$ & $1.02^{c} \pm 0.03$ & $9.07^{c} \pm 0.42$ & $564.6^{c} \pm 1.52$ & $652^{\mathrm{c}} \pm 2.66$ & $1.15^{\mathrm{d}} \pm 0.00$ \\
\hline & $4^{\text {th }}$ & $610.8^{a} \pm 1.77$ & $1.03^{c} \pm 0.03$ & $14.85^{\mathrm{b}} \pm 0.58$ & $629.5^{\mathrm{b}} \pm 2.20$ & $1057^{\mathrm{b}} \pm 4.91$ & $1.68^{\mathrm{c}} \pm 0.15$ \\
\hline & $5^{\text {th }}$ & $566.7^{\mathrm{b}} \pm 2.12$ & $1.37^{b} \pm 0.04$ & $15.41^{\mathrm{a}} \pm 0.77$ & $772.5^{a} \pm 1.33$ & $1853^{a} \pm 2.75$ & $2.39^{a} \pm 0.01$ \\
\hline$P$ value & & 0.000 & 0.002 & 0.009 & 0.002 & 0.025 & 0.001 \\
\hline
\end{tabular}

Means carrying different superscripts in the same column are significantly different at $(\mathrm{P} \leq 0.05)$ or highly significantly different at $(\mathrm{P}<0.01)$. Means carrying the same superscripts in the same column are non-significantly different at $(\mathrm{P}<0.05)$.

BWG=Weight Gain, FI=Feed Intake, FCR=Feed Conversion Ratio, and PI=Performance Index, WI=Water intake, WI/FI=Water to feed intake ratio, $\mathrm{SE}=\mathrm{Standard}$ error. 
Table 3: Live and carcass quality characteristics (Mean \pm SE) of broilers raised on different housing floor systems.

\begin{tabular}{|c|c|c|c|c|c|c|}
\hline \multirow[t]{2}{*}{ Floor system } & \multirow{2}{*}{$\mathrm{LBW} / \mathrm{g}$} & \multirow{2}{*}{$\mathrm{CW} / \mathrm{g}$} & \multicolumn{4}{|c|}{ Edible and immune organs weight } \\
\hline & & & Liver/g & Spleen / g & Heart / $g$ & Bursa / g \\
\hline \multicolumn{7}{|c|}{ Overall means among different floor systems } \\
\hline Wood shaving & $1646.5^{\mathrm{d}} \pm 3.88$ & $1239.8^{c} \pm 6.73$ & $42.1^{\mathrm{c}} \pm 0.91$ & $1.64^{c} \pm 0.03$ & $9.52^{c} \pm 0.18$ & $1.02^{c} \pm 0.01$ \\
\hline Rice husks & $1660.3^{\mathrm{d}} \pm 2.17$ & $1207.4^{\mathrm{d}} \pm 5.87$ & $39.6^{\mathrm{d}} \pm 0.24$ & $1.64^{c} \pm 0.02$ & $9.22^{c} \pm 0.17$ & $1.00^{c} \pm 0.01$ \\
\hline Wheat straw & $1762.8^{c} \pm 3.23$ & $1210.0^{\mathrm{d}} \pm 3.72$ & $43.7^{c} \pm 0.48$ & $1.69^{c} \pm 0.04$ & $9.66^{c} \pm 0.17$ & $1.06^{c} \pm 0.02$ \\
\hline Slats & $2111.4^{b} \pm 5.28$ & $1840.1^{\mathrm{b}} \pm 3.23$ & $55.6^{\mathrm{b}} \pm 0.26$ & $3.47^{b} \pm 0.05$ & $15.93^{b} \pm 0.18$ & $1.48^{\mathrm{b}} \pm 0.03$ \\
\hline Battery & $2207.0^{\mathrm{a}} \pm 4.13$ & $1874.8^{\mathrm{a}} \pm 5.72$ & $59.6^{a} \pm 0.35$ & $3.67^{\mathrm{a}} \pm 0.05$ & $19.84^{\mathrm{a}} \pm 0.19$ & $1.59^{\mathrm{a}} \pm 0.03$ \\
\hline $\mathrm{P}$ value & 0.000 & 0.001 & 0.000 & 0.001 & 0.000 & 0.000 \\
\hline
\end{tabular}

Means carrying different superscripts in the same column are significantly different at $(\mathrm{P} \leq 0.05)$ or highly significantly different at $(\mathrm{P}<0.01)$. Means carrying the same superscripts in the same column are non-significantly different at $(\mathrm{P}<0.05)$.

LBW=Live body weight, CW=Carcass weight, $\mathrm{SE}=$ Standard error.

Table 4: Stress and antioxidant markers (Mean \pm SE) of broilers raised on different housing floor systems.

\begin{tabular}{|c|c|c|c|c|c|}
\hline Floor system & $\begin{array}{l}\text { Cort } \\
\text { mcg.dl }{ }^{-1}\end{array}$ & $\begin{array}{l}\text { TAC } \\
\text { mM.L }{ }^{-1}\end{array}$ & $\begin{array}{l}\text { LDH } \\
\text { IU.L }^{-1}\end{array}$ & $\begin{array}{l}\text { MDA } \\
\text { nmol.ml }\end{array}$ & $\begin{array}{l}\text { SOD } \\
\text { U.ml-1 }\end{array}$ \\
\hline \multicolumn{6}{|c|}{ Overall means among different floor systems } \\
\hline Wood shaving & $25.4^{\mathrm{a}} \pm 0.03$ & $2.32^{\mathrm{a}} \pm 0.00$ & $374.6^{a} \pm 2.40$ & $37.1^{\mathrm{a}} \pm 0.25$ & $310.6^{a} \pm 2.95$ \\
\hline Rice husks & $20.9^{\mathrm{b}} \pm 0.02$ & $2.21^{\mathrm{b}} \pm 0.00$ & $341.0^{\mathrm{b}} \pm 0.89$ & $40.4^{\mathrm{a}} \pm 0.26$ & $288.9^{b} \pm 0.54$ \\
\hline Wheat straw & $16.1^{\mathrm{c}} \pm 0.02$ & $1.83^{c} \pm 0.01$ & $282.1^{\mathrm{c}} \pm 0.89$ & $23.6^{\mathrm{b}} \pm 0.14$ & $226.9^{c} \pm 1.27$ \\
\hline Slats & $5.6^{\mathrm{d}} \pm 0.03$ & $0.99^{\mathrm{d}} \pm 0.01$ & $240.9^{\mathrm{d}} \pm 0.91$ & $20.2^{\mathrm{b}} \pm 0.16$ & $197.7^{\mathrm{d}} \pm 0.63$ \\
\hline Battery & $5.2^{\mathrm{d}} \pm 0.02$ & $0.59^{\mathrm{e}} \pm 0.01$ & $205.3^{\mathrm{e}} \pm 0.88$ & $15.9^{\mathrm{b}} \pm 0.33$ & $150.6^{\mathrm{e}} \pm 0.89$ \\
\hline $\mathrm{P}$ value & 0.000 & 0.001 & 0.000 & 0.007 & 0.000 \\
\hline
\end{tabular}

Means carrying different superscripts in the same column are significantly different at $(\mathrm{P} \leq 0.05)$ or highly significantly different at $(\mathrm{P}<0.01)$. Means carrying the same superscripts in the same column are non-significantly different at $(\mathrm{P}<0.05)$.

Cort=Cortisol hormone, $\mathrm{TAC}=$ Total antioxidant capacity, $\mathrm{LDH}=$ Lactate dehydrogenase, $\mathrm{MDA}=$ Malondialdehyde, $\mathrm{SOD}=$ Superoxide dismutase, $\mathrm{SE}=\mathrm{Standard}$ error.

Table 5: Immunoglobulin concentrations (Mean $\pm \mathrm{SE}$ ) of broilers raised on different housing floor systems.

\begin{tabular}{|c|c|c|}
\hline Floor system & $\begin{array}{l}\text { IgG } \\
\text { mg.dl }\end{array}$ & $\begin{array}{l}\text { IgM } \\
\text { mg.dl }\end{array}$ \\
\hline \multicolumn{3}{|c|}{ Overall means among different floor systems } \\
\hline Wood shaving & $1351.2^{\mathrm{e}} \pm 2.39$ & $360.0^{\mathrm{e}} \pm 0.98$ \\
\hline Rice husks & $1423.2^{\mathrm{d}} \pm 1.61$ & $389.1^{\mathrm{d}} \pm 1.09$ \\
\hline Wheat straw & $1587.0^{c} \pm 1.58$ & $461.6^{c} \pm 1.64$ \\
\hline Slats & $1674.3^{b} \pm 2.68$ & $532.3^{\mathrm{b}} \pm 1.47$ \\
\hline Battery & $1863.0^{a} \pm 2.28$ & $582.1^{\mathrm{a}} \pm 1.42$ \\
\hline$P$ value & 0.000 & 0.001 \\
\hline
\end{tabular}

Means carrying different superscripts in the same column are significantly different at $(\mathrm{P} \leq 0.05)$ or highly significantly different at $(\mathrm{P}<0.01)$. Means carrying the same superscripts in the same column are non-significantly different at $(\mathrm{P}<0.05)$.

IgG=Immunoglobulin G, IgM=Immunoglobulin M, SE=Standard error.

shaving and rice husks, and in total bacterial counts of air samples in battery and on slatted floors with no significant differences in total bacterial counts of the two systems.

Highly significant declines $(\mathrm{P}<0.01)$ were recorded as shown in Table 7 in total Enterobacteriaceae counts of intestinal (duodenal); floor swabs; waterers swabs and air samples in broilers raised in battery and on slatted floors, in total Enterobacteriaceae counts of wall swabs in battery and on slatted floors with no significant differences in total Enterobacteriaceae counts between wheat straw and rice husks, and total Enterobacteriaceae counts of feeder swabs in battery and on slatted floors with no significant differences in total Enterobacteriaceae counts between deep litter systems. 
Table 6: Total bacterial counts of environmental and non-environmental samples (Mean CFU/ml \pm SE) collected from broiler rooms of different housing floor systems.

\begin{tabular}{lllllll} 
Floor system & \multicolumn{2}{l}{ Intestinal swabs } & \multicolumn{2}{l}{ Environmental swabs } & \multicolumn{2}{l}{ Air } \\
& & \multicolumn{2}{l}{ Floor } & Wall & Feeders & Waterer \\
Overall means among different floor systems & & & & & \\
Wood shaving & $4.58^{\mathrm{b}} \pm 0.02$ & $5.25^{\mathrm{a}} \pm 0.00$ & $5.05^{\mathrm{a}} \pm 0.00$ & $4.90^{\mathrm{a}} \pm 0.01$ & $4.89^{\mathrm{a}} \pm 0.02$ & $3.73^{\mathrm{a}} \pm 0.00$ \\
Rice husks & $4.40^{\mathrm{c}} \pm 0.01$ & $4.96^{\mathrm{b}} \pm 0.00$ & $4.93^{\mathrm{b}} \pm 0.01$ & $4.42^{\mathrm{b}} \pm 0.01$ & $4.96^{\mathrm{a}} \pm 0.00$ & $3.10^{\mathrm{b}} \pm 0.01$ \\
Wheat straw & $5.06^{\mathrm{a}} \pm 0.09$ & $4.79^{\mathrm{c}} \pm 0.02$ & $4.71^{\mathrm{c}} \pm 0.05$ & $4.89^{\mathrm{a}} \pm 0.10$ & $3.81^{\mathrm{d}} \pm 0.05$ & $2.72^{\mathrm{c}} \pm 0.03$ \\
Slats & $4.35^{\mathrm{c}} \pm 0.01$ & $4.81^{\mathrm{c}} \pm 0.01$ & $4.52^{\mathrm{d}} \pm 0.01$ & $4.19^{\mathrm{c}} \pm 0.01$ & $4.35^{\mathrm{b}} \pm 0.01$ & $2.11^{\mathrm{d}} \pm 0.01$ \\
Battery & $4.22^{\mathrm{e}} \pm 0.01$ & $4.14^{\mathrm{d}} \pm 0.01$ & $4.21^{\mathrm{e}} \pm 0.01$ & $3.92^{\mathrm{d}} \pm 0.01$ & $4.13^{\mathrm{c}} \pm 0.01$ & $2.11^{\mathrm{d}} \pm 0.01$ \\
P value & 0.002 & 0.001 & 0.000 & 0.002 & 0.001 & 0.002
\end{tabular}

Means carrying different superscripts in the same column are significantly different at $(\mathrm{P} \leq 0.05)$ or highly significantly different at $(\mathrm{P}<0.01)$. Means carrying the same superscripts in the same column are non-significantly different at $(\mathrm{P}<0.05)$.

$\mathrm{CFU}=$ Colony forming unit, $\mathrm{SE}=\mathrm{Standard}$ error.

Table 7: Total Enterobacteriaceae counts of environmental and non-environmental samples (Mean CFU/ml $\pm \mathrm{SE}$ ) collected from broiler rooms of different housing floor systems.

\begin{tabular}{lllllll} 
Floor system & \multicolumn{1}{c}{ Intestinal swabs } & \multicolumn{2}{l}{ Environmental swabs } & \multicolumn{2}{l}{ Air } \\
& & Floor & Wall & Feeders & Waterer & \\
Overall means among different floor systems & & & & & \\
Wood shaving & $3.42^{\mathrm{a}} \pm 0.02$ & $3.74^{\mathrm{a}} \pm 0.02$ & $3.34^{\mathrm{a}} \pm 0.02$ & $3.19^{\mathrm{a}} \pm 0.02$ & $3.20^{\mathrm{a}} \pm 0.02$ & $2.72^{\mathrm{a}} \pm 0.02$ \\
Rice husks & $3.11^{\mathrm{b}} \pm 0.02$ & $3.05^{\mathrm{b}} \pm 0.02$ & $3.08^{\mathrm{b}} \pm 0.02$ & $3.19^{\mathrm{a}} \pm 0.02$ & $3.11^{\mathrm{b}} \pm 0.02$ & $2.42^{\mathrm{b}} \pm 0.02$ \\
Wheat straw & $2.77^{\mathrm{c}} \pm 0.02$ & $2.75^{\mathrm{c}} \pm 0.06$ & $3.01^{\mathrm{b}} \pm 0.05$ & $3.25^{\mathrm{a}} \pm 0.04$ & $2.98^{\mathrm{c}} \pm 0.02$ & $2.08^{\mathrm{c}} \pm 0.02$ \\
Slats & $2.26^{\mathrm{d}} \pm 0.03$ & $2.27^{\mathrm{d}} \pm 0.02$ & $2.18^{\mathrm{c}} \pm 0.02$ & $2.16^{ \pm} \pm 0.02$ & $2.30^{\mathrm{d}} \pm 0.02$ & $1.55^{\mathrm{d}} \pm 0.03$ \\
Battery & $2.15^{\mathrm{e}} \pm 0.02$ & $1.80^{\mathrm{e}} \pm 0.01$ & $1.97^{\mathrm{d}} \pm 0.00$ & $1.75^{\mathrm{c}} \pm 0.01$ & $1.65^{\mathrm{e}} \pm 0.02$ & $1.45^{\mathrm{e}} \pm 0.02$ \\
P value & 0.000 & 0.000 & 0.001 & 0.002 & 0.000 & 0.000
\end{tabular}

Means carrying different superscripts in the same column are significantly different at $(\mathrm{P} \leq 0.05)$ or highly significantly different at $(\mathrm{P}<0.01)$. Means carrying the same superscripts in the same column are non-significantly different at $(\mathrm{P}<0.05)$.

$\mathrm{CFU}=$ Colony forming unit, $\mathrm{SE}=$ Standard error.

\section{DISCUSSION}

Feeding regimens and housing system represented the most important factors that control commercial poultry production. Poultry industry worldwide relayed for centuries mainly on deep litter housing systems, as litter usually provided broilers with more comfortable conditions and influence the financial outcomes. Good litter materials should maintain indoor temperature, absorb extra moisture, help to minimize the volatilization of ammonia gas into microclimatic air, and resist the multiplication of many pathogenic microorganisms. Litter witnessed the usage of many materials like rice husk and wheat straw as experimented by Sreehari and Sharma, (2010), soybean straw as recommended by De Avila et al. (2008), gypsum that was tested and recommended by Grimes et al. (2006), and sand as reported by Shields et al. (2005).

The current results recorded that the usage of wood shaving, rice husks, and wheat straw litters compared to slats and cage systems contributed to higher levels of carbon dioxide and ammonia gases in the microclimatic air de- veloping some conditions that might contribute to the development of many diseases. The results were consistent to those reported by Lin et al. (2017), Pereira et al. (2017) and Naseem and King, (2018) who agreed that litter material undergoes decomposition and fermentation under the influence of many factors like litter management factors, microclimatic temperature, humidity, and ventilation rate contributed to increases in the concentration of carbon dioxide, ammonia, nitrous oxide, and methane. The finding of the present study were consistent with those reported by Jugowar et al. (2017). They recorded an increase in the levels of carbon dioxide and ammonia gases, and they attributed this increase to the decomposition of litter material in the presence of significantly lower ventilation rates especially in cold months of the year. Méda et al. (2011) recorded similar findings to the current results; increases in ammonia volatilization due to increased litter moisture content was a result of increases in microclimatic relative humidity.

The poultry production witnessed great expansion, and within this expansion, the interest in using other bedding 
materials as slats and cages have been increased as they contributed fewer problems and enhanced poultry welfare. The most common recorded problems from using litter were footpad lesions, hock joint dermatitis, hock joint arthritis, and femoral head necrosis as reported by Dawkins et al. (2017). Çavuşoğlu et al. (2018) also investigated some welfare parameters in 210-day-old male broiler chickens raised on deep litter, a mix of litter and slat system, and a slatted floor, they recorded negative influences on the measured welfare parameters in broilers raised on deep-litter, while the high live body weights recorded in broilers raised on slat floor contributed to severe pathological affections of the breast and shoulder.

Meanwhile, Adam (2017) reported that a deep litter housing system provided an optimum environment for layers and egg quality in the presence of good managerial procedures compared to battery cages housing system in open-sided houses. Kaukonen et al. (2016) stated that litter abiotic conditions are the keystone for the development of many pathological conditions like footpad dermatitis, hock burns, and breast blisters. They also concluded that maintaining litter quality is not enough to minimize the incidence of these pathological affections.

The current research reported significantly higher weight gains, performance indices, and live body weights, as well as, lower feed intake and consequently lower and enhanced feed conversion ratio in broilers raised in slat and cage systems compared to deep litter systems. The results were consistent with those recorded by Dhaliwal et al. (2018) and Davasgaium and Boodoo, (2000) who revealed that rice husk, wheat straw, and the mustard stalk were more or less similar, although birds on saw consumed the lowest amount of feed. Al-Bahouh et al. (2012) and Santos et al. (2008) reported enhanced performance of broiler breeds (Indian River ${ }^{\circledast}, \mathrm{Cobb}^{\circledR} 500$, and Ross ${ }^{\circledR}$ 308) that were raised in cages than on floors. Chuppava et al. (2018) conducted an experimental trial using Ross ${ }^{\circledR} 308$ broiler breed to investigate the influence of different flooring systems $(50 \%$ or $100 \%$ slatted floors) with reduced contact to the excreta on body weight, carcass weight, feed intake, they reported that final body weight was significantly increased by the end of the fattening cycle which lasts for day 36 by using fully-slatted floors compared to commonly littered floors. Broilers on the plastic slatted floor were observed to have relatively higher weight gains and higher feed conversion ratios than when raised on wood shavings, this may be attributed to the prohibited possibilities for the birds to peck and manipulate particles in case of a slatted floor, so feed pecking occurs rather than the slatted floor resulting in higher feed intake. Pereira et al. (2007) observed that the presence of air movement between the manure and perforated floor can reduce heat stress in the slat-floor system, which in turn increases the productivity of birds.

Samli et al. (2010) explained that advanced floor housing as cages can adventurously and positively influence the broiler's performance and intestinal microbiota. On the other hand, Fouad et al. (2008) reported that chicks housed on wood shaving were more active, had higher metabolic rates, and increased walking and playing behavior (mixing of floor materials). While, Pedroso et al. (2006) recorded no differences in feed intakes and gain ratios neither on the floor nor on cages for broilers. Darwish et al. (2017) found that Evian broiler on litter floor had higher body weights, weight gains, and feed consumption in most of the studied intervals compared to those raised in battery cages ( $p \leq 0.05$ and 0.01 ), and no significant differences for feed conversion ratios, carcass characteristics, some blood parameters, and some immunity between the littered floor and battery cage systems.

The current results reported declined carcass and organs' weights like liver, heart, spleen, and bursa of Fabricius in broilers raised on the tested deep litter systems including wood shavings, rice husk, and wheat straw. The results were consistent with those reported by Dal Bosco et al. (2015) who concluded that housing broilers on a deep litter system contributed to lower meat $\mathrm{pH}$ values and color, as well as, the higher percentage of monosaturated fatty acids and long-chain polyunsaturated fatty acids. Also, Darwish et al. (2017) reported higher performance and increased carcass yield in Evian broilers raised on floor system in comparison with battery cages. Li et al. (2017) recorded no significant differences in productive performances and the incidence of pathological affections development in broilers raised on the conventional deep litter system and those raised plastic slats.

Our results revealed significant increases in serum concentrations of total immunoglobulin IgG and IgM in broilers raised in the battery system and on the slatted floor. The result was consistent to those of Taherparvar et al. (2019) who investigated the influence of litter (sand, wood shaving, and paper) on some blood parameters and immunity in 270 day-old male Ross ${ }^{\circledR} 308$ broiler chicken, they recorded significant increases in total immunoglobulin titer contributed to the significant increase in immunoglobulin $\mathrm{M}$. The current results recorded a significant decline in total bacterial counts of intestinal (duodenal) swabs in broilers raised in battery and on slatted floors compared to the deep litter systems. The current results might be attributed to the maintaining action of strict hygienic measures on the slatted floor and battery cages compared to deep litter. De Almeida et al. (2017) concluded that using slat-floor systems can be more efficient and contribute to lower pathological affections, high-quality environment, and high live 
body weights. De Jong et al. (2016) who reported a necessity to maintain hygienic measure and management of floor system to maintain the optimum welfare conditions in broilers. They also found that the welfare parameters like cleanliness, hock burn, footpad dermatitis, and gait score on-farm are highly correlated with the development of hock burn and footpad dermatitis in a slaughter plant.

\section{CONCLUSION}

The study tested different types of floor systems that can be used in broiler farms like wood shaving, rice husks, wheat straw, slats, and cages. The study revealed that despite the numerous advantages of the deep litter systems and their enhancing influence over growth traits and immunity, they still carry dangerous disadvantages when mishandled, these disadvantages can be summarized in creating friendly conditions for the growth and multiplication of many pathogenic micro-organisms and contribute to higher chances of disease development.

Slatted floors and battery cages were able to maintain indoor air quality (levels of carbon dioxide and ammonia), reduce microbial contamination by maintaining a harsh condition that obstacle the growth and multiplication of pathogenic microorganisms, and enhance growth traits and immunity of broiler chickens.

\section{ACKNOWLEDGMENT}

Sincere thanking for Prof. M.A. Sobieh for his generous directions during the experiment.

\section{CONFLiCT OF INTEREST}

The author declared no conflict of interest.

\section{ETHical Approval}

The procedures used in the present study were approved by the Scientific Research Ethics Committee on animal and poultry researches, Faculty of Veterinary Medicine, Suez Canal University, Egypt (approval number 2020012).

\section{AUTHORS' CONTRIBUTIONS}

ESS designed the experiment, Participated in preparation, executing the experiment, and in writing the manuscript. $\mathrm{RAH}$ participated in executing the experiment and in writing the manuscript.

\section{REFERENCES}

-Abreu VMN, Abreu PG, Jaenisch FRF, Coldebella A, Paiva DP (2011). Effect of floor type (dirt or concrete) on litter quality, house environmental conditions, and performance of broilers. Rev. Bras. Cienc. Avic. 13(2): 127-137. https://doi. org/10.1590/S1516-635X2011000200007

-Adam IY (2017). Effect of deep-litter and battery cages system on the productive performance of commercial layers in Elobied, Sudan. Master Thesis, Department of Animal Production, Faculty of Natural Resources and Environmental Studies, University of Kordofan, Sudan.

-Al-Bahouh ME, Al-Nasser AY, Abdullah FK, Ragheb G, Mashaly MM (2012). Production performance of different broiler breeds under different housing system. Int.J.Poult. Sci. 11(3): 190-195. https://doi.org/10.3923/ijps.2012.190.195

-American Public Health Association, American Water Works Association, Water Environment Federation (2017). Standard methods for the examination of water and wastewater. By EW Rice, RB Baird, AD Eaton, American Water Work Association Publications $23^{\text {rd }}$ Ed, Washington D.C.

-American Public Health Association, American Water Works Association, Water Environment Federation (2012). Standard methods for the examination of water and wastewater. American Water Work Association Publications 2012; 22th Ed, Washington D.C.

-Applegate TJ, Angel R (2014). Nutrient requirements of poultry publication: History and need for an update. J. Appl. Poult. Res. 23(3): 567-575. https://doi.org/10.3382/japr.201400980

-Aviagen (2016). A guide to managing broilers in opensided housing. Available at:http://en.aviagen.com/assets/ Tech_center/Broiler_Breeder_Tech_Articles/English/ AVIAEnvMgtOpenSidedHseBroiler-EN-2016.pdf.

-Bilal K, Mehmood S, Akram M, Imran S, Sahota AW, Javed K, Hussain J, Ashfaq A (2014). Growth performance of broilers under two rearing systems in three different housing zones in an environmentally controlled house during winter. J. Anim. Plant Sci. 24, 1039-1044, 2014.

- Çavuşoğlu E, Petek M, Abdourhamane IM, Akkoc A, Topal E (2018). Effect of different floor housing system on the welfare of fast-growing broilers with an extended fattening period. Arch. Anim. Breed. 61:9-16. https://doi.org/10.5194/aab61-9-2018

- Chuppava B, Visscher C, Kamphues J (2018). Effect of different flooring design on the performance and foot pad health in broilers and turkeys. Anim. 8(5): 70. https://doi. org/10.3390/ani8050070

-Dal Bosco A, Szendrő SZ, Matics ZS, Castellini C, Ruggeri S, Szendrő k, Martino M, Mattioli S, Dalle Zotte A, Gerencser ZS (2015). Effect of floor type on carcass and meat quality of pen raised growing rabbits. World Rabbit Sci. 23: 19-26. https://doi.org/10.4995/wrs.2015.3270

-Darwish AH, El-Sayiad GhA, El-Maghawry AM, Mahrose KhM (2017). Growth performance, carcass traits and some blood parameters of broiler chicks as affected by housing system. Zagazig J. Agric. Res. 44(4): 1379-1387. https://doi. org/10.21608/zjar.2017.52941

-Davasgaium MM, Boodoo AA (2000). Use of bagasse as a potential source of litter material for broiler production. Internet address: http://farc.gov.mu/amas97/html/p18txt. htm.

-Dawkins MS, Roberts SJ, Cain RJ, Nickson T, Don-nelly CA (2017). Early warning of footpad dermatitis and hock burn in broiler chicken flocks using optical flow, body weight and water consumption. Vet. Rec. 11: 1-5. 
- De Almeida EA, Arantes de Souza LF, Sant'Anna AC, Bahiense RN, Macari M, Furlan RL (2017). Poultry rearing on perforated plastic floors and the effect on air quality, growth performance, and carcass injuries-Experiment 1: Thermal comfort. Poult. Sci. 96, 3155-3162. https://doi.org/10.3382/ ps/pex131

-De Avila VS, Oliveira U, Figueiredo EAP, Costa CAF, Abreu VMN, Rosa PS (2008). Evaluation of alternative materials to replace shavings such as poultry litter. Rev. Bras. Zootecn 37: 273-277. https://doi.org/10.1590/S151635982008000200013

-De Jong IC, Gunnink H (2014). Wet litter not only induces footpad dermatitis but also reduces overall welfare, technical performance, and carcass yield in broiler chickens. J. Appl. Poult. Res. 23, 51-58. https://doi.org/10.3382/japr.201300803

-De Jong IC, Hindle VA, Butterworth A, Engel B, Ferrari P, Gunnink H, Perez Moya T, Tuyttens FAM, Van Reenen CG (2016). Simplifying the Welfare Quality ${ }^{\circledR}$ assessment protocol for broiler chicken welfare. Anim. 10(1): 117-127 https://doi.org/10.1017/S1751731115001706.

-Dhaliwal APS, Dhillon BS, Brar JS (2018). Evaluation of different litter materials for broiler production. Int. J. Curr. Microbiol. Appl. Sci. 7(12): 1041-1045. https://doi. org/10.20546/ijcmas.2018.712.130

- Fouad MA, Abdel Razek AH, Badawy El, Sayed M (2008). Broilers welfare and economics under two management alternatives on commercial scale. Int. J. Poult. Sci. 7: 11671173. https://doi.org/10.3923/ijps.2008.1167.1173

- Garcia RG, Almeida Paz ICL, Caldara FR, Nääs IA, Pereira DF, Ferreira VMOS (2012). Selecting the most adequate bedding material for broiler production in Brazil. Rev. Bras. Cienc. Avic. 14(2): 121-127. https://doi.org/10.1590/ S1516-635X2012000200006

- Grimes JL, Carter TA, Godwin JL (2006). Use of a litter material made from cotton waste, gypsum, and old newsprint for rearing broiler chickens. Poult. Sci. 85: 563-568. https://doi. org/10.1093/ps/85.3.563

-Jacob FG, Baracho MS, Nääs IA, Lima NSD, Salgado DD, Souza R (2016). Risk of incidence of hock burn and pododermatitis in broilers reared under commercial conditions. Rev. Bras. Cienc. Avic. 18, 357-362. https://doi. org/10.1590/1806-9061-2015-0183

-Jugowar JL, Rzeźnik W, Mielcarek P (2017). Emission of ammonia, nitrous oxide and methane from hen house in deep litter/slatted floor system. J. Res. Apps. Agric. Engine. 62, 64-69.

-Kaukonen E, Norring M, Valros A (2016). Effect of litter quality on foot pad dermatitis, hock burns and breast blisters in broiler breeders during the production period. Avian Pathol. 45 (6): 667-673. https://doi.org/10.1080/03079457.2016.1 197377

-Kim SK, Lee JH (2016). Biofilm modeling systems. Korean J. Microbiol. 52(2): 125-139. https://doi.org/10.7845/ kjm.2016.6027

-Li H, Wen X, Alphin R, Zhu Z, Zhou Z (2017). Effect of two different broiler flooring systems on production performances, welfare, and environment under commercial production conditions. Poult. Sci. 96: 1108-1119. https:// doi.org/10.3382/ps/pew440

-Lin X,Zhang R, Jiang S, El-Mashad H,Xin H.(2017).Emissions of ammonia, carbon dioxide and particulate matter from cage-free layer houses in California. Atmos. Environ. 152:
246-255. https://doi.org/10.1016/j.atmosenv.2016.12.018

- Méda B, Hassouna M, Aubert C, Robin P, Dourmad JY (2011). Influence of rearing conditions and manure management practices on ammonia and greenhouse gas emissions from poultry houses. World's Poult. Sci. 67, 441-456. https://doi. org/10.1017/S0043933911000493

- Murray PR, Rosenthal KS, Pfaller MA (2015). Medical Microbiology, 8th Edition, Elsevier Health Sciences, Philadelphia, PA, USA.

- Naseem S, King AJ (2018). Ammonia production in poultry houses can affect health of humans, birds, and the environment-techniques for its reduction during poultry production. Environ. Sci. Pollut. Res. 25: 15269-15293. https://doi.org/10.1007/s11356-018-2018-y

- National Research Council (NRC) (1994). Nutrient requirements for poultry. Ninth revised edition, 1994, National Research Council, New York.

- Özhan N, Simsek UC, Ozcelik M (2016). Comparison of floor and cage housing systems in terms of some welfare assessments in broiler. Kafkas Univ. Vet. Fak. 63: 317-322. https://doi.org/10.1501/Vetfak_0000002746

- Pedroso AA, Menten JF, Lambais MR, Racanicci AMC, Longo FA, Sorbara JOB (2006). Intestinal bacterial community and growth performance of chickens fed diets containing antibiotics. Poult. Sci. 85(4): 747-752 https://doi. org/10.1093/ps/85.4.747

- Pereira DF, Nääs I, Romanini C, Salgado D, Pereira G (2007). Broiler breeder behavior and egg production as function of environmental temperature. Rev. Bras. Cienc. Avic. 9: 9-16 https://doi.org/10.1590/S1516-635X2007000100002.

- Pereira JLS, Ferreira S, Garcia CSP, Conde A, Ferreira P, Pinheiro V, Trindade H (2017). Assessment of ammonia and carbon dioxide concentrations in a breeding hen building under Portuguese winter. Int. J. Food Biosys. Engine. 5: 1-6.

-Petek M, Çavusoglu E, Topal E, Ünal C, Ab-dourhamane IM (2015). Effects of slatted floor housing on animal welfare in broiler production, 3rd International Poultry Meat Congress, 22-26 April 2015, Antalya, Turkey, Proceedings.

-Petek M, Ustüner H, Yesilbag D (2014). Effects of stocking density and litter type on litter quality and growth performance of broiler chicken. Kafkas Univ. Vet. Fak. 20, 743-748. https://doi.org/10.9775/kvfd.2014.11016

-Samli HE, Dezcan S, Koc F, Ozduven ML, Agma Okur A, Senkoylu N (2010). Effects of Enterococcus faecium supplementation and floor type on performance, morphology of erythrocytes and intestinal microbiota in broiler chickens. British Poult. Sci. 51 (4): 564-568. https://doi.org/10.1080/ 00071668.2010.507241

- Santos FBO, Sheldon BW, Santos Jr AA, Ferket PR (2008). Influence of housing system, grain type and particle size on Salmonella colonization and shedding of broiler fed triticale or corn-soybean meal diets. Poult. Sci. 87: 833-838. https:// doi.org/10.3382/ps.2006-00417

- Shields SJ, Garner JP, Mench JA (2005). Effect of sand and wood-shavings bedding on the behavior of broiler chickens. Poult. Sci. 84: 1816-1824. https://doi.org/10.1093/ ps/84.12.1816

- Sohirat Torfy M, Mirzadeh KH, Tabatabaei Vakili S, Chaji M (2017). Effects of different levels of nanoselenium solution in water on performance, ileum microbial populations, tibia bone parameters, litter quality and some blood parameters of broiler chickens. J. Anim. Sci. Res. 26(4): 189-211.

-Soliman ES, Abdallah MS (2020). Assessment of biosecurity 
measures in broiler's farms in the Suez Canal area - Egypt using a seasonal prevalence of Salmonellosis.Vet.World 13(4): 622-632. https://doi.org/10.14202/vetworld.2020.622-632

-Soliman ES, Hamad RT, Ahmed A (2017). Prophylactic and immune modulatory influences of Nigella sativa Linn. in broilers exposed to biological challenge. Vet. World 10(12):1447-1455. https://doi.org/10.14202/ vetworld.2017.1447-1455

- Soliman ES, Hassan RA (2017). Evaluation of superphosphate and meta-bisulfide efficiency in litter treatment on productive performance and immunity of broilers exposed to ammonia stress. Adv. Anim. Vet. Sci. 5(6): 253-259.

-Soliman ES, Hassan RA (2019). Impact of lighting color and duration on productive performance and Newcastle disease vaccination efficiency in broiler chickens. Vet. World 12(7): 1052-1059. https://doi.org/10.14202/ vetworld.2019.1052-1059

-Soliman ES, Moawed SA, Ziaan AMG (2016). Assessing cleaning and disinfection regime in a slaughterhouse against carcasses contamination. Adv. Anim. Vet. Sci. 4(9): 449-457 https://doi.org/10.14737/journal.aavs/2016/4.9.449.457.
-Soliman ES, Sallam NH,Abouelhassan EM(2018).Effectiveness of poultry litter amendments on bacterial survival and Eimeria oocyst sporulation. Vet. World 11(8): 1064-1073. https://doi.org/10.14202/vetworld.2018.1064-1073

-SPSS (2016). Statistical Packages of Social Sciences. Version 21 for windows. SPSS. Inc. USA

-Sreehari S, Sharma RK (2010). Effect of litter type and stocking density on the performance of broilers. Indian J. Poult. Sci. 45 (1): 105-107.

-Taherparvar G, Seidavi AR, Asadpour L, Payan-Carreira R (2019). Impact of litter and litter amendments on blood variables and immunity of broiler chickens. J. Anim. Sci. 29(2): 119-132.

-Thrusfield M. (2005). Veterinary epidemiology, 3rd ed. Oxford: Wiley Blackwell. 228-330.

-Wu YN, Yan FF, Hu JY, Chen H, Tucker CM, Green AR, Cheng HW (2017). The effect of chronic ammonia exposure on acute-phase proteins, immunoglobulin, and cytokines in laying hens. Poult. Sci. 96(6):1524-1530. https://doi. org/10.3382/ps/pew454 